\title{
Green synthesis, characterization and catalytic activity of palladium nanoparticles by xanthan gum
}

\author{
Amrutham Santoshi kumari • Maragoni Venkatesham • \\ Dasari Ayodhya · Guttena Veerabhadram
}

Received: 24 April 2014/ Accepted: 7 May 2014/Published online: 31 May 2014

(C) The Author(s) 2014. This article is published with open access at Springerlink.com

\begin{abstract}
Here, we report the synthesis, characterization and catalytic evaluation of palladium nanoparticles (PdNPs) using xanthan gum, acting as both reducing and stabilizing agent without using any synthetic reagent. The uniqueness of our method lies in its fast synthesis rates using hydrothermal method in autoclave at a pressure of $15 \mathrm{psi}$ and at $120{ }^{\circ} \mathrm{C}$ temperature by $10 \mathrm{~min}$ time. The formation and size of the PdNPs were characterized by UV-visible spectroscopy, X-ray diffraction, Fourier transform infrared spectroscopy and transmission electron microscopy. The catalytic activity of PdNPs was evaluated on the reduction of 4-nitrophenol to 4-aminophenol by sodium borohydride using spectrophotometry.
\end{abstract}

Keywords Autoclave - Catalytic activity - 4-Nitrophenol · Palladium nanoparticle $\cdot$ Xanthan gum

\section{Introduction}

In the last decade, the importance of understanding of science at the nanometre scale has attracted the interest of number of groups all over the world and has led to the emergence of a new interdisciplinary field called nanoscience, because of the size-dependent optical, electronic, and catalytic properties of nanoparticles (Prashant et al. 2006), and even in biological and medical science applications (Salata 2004). There are enormous changes in physical and chemical properties, both quantitatively and qualitatively,

A. Santoshi kumari - M. Venkatesham - D. Ayodhya ·

G. Veerabhadram $(\bowtie)$

Department of Chemistry, University College of Science,

Osmania University, Hyderabad 500007, India

e-mail: gvbhadram@gmail.com from those of bulk materials as these materials are derived by manipulation at the atomic or molecular levels and because of surface to volume ratio and quantum size effect (Roucoux et al. 2002; Rosi and Mirkin 2005; Yang et al. 2006). Palladium nanoparticles are of great interest owing to their application both in heterogeneous and homogeneous catalyses, their high surface-to-volume ratio and high surface energy (Narayanan and El-Sayed 2005). In addition, Pd is exploited as a catalyst in various coupling reactions like Heck coupling (Karimi and Enders 2006), Suzuki coupling (Klingensmith and Leadbeater 2003), and hydrogenation of allyl alcohols (Wilson et al. 2006). Till now, production of PdNPs involved different reducing chemicals such as $\mathrm{NaBH}_{4}$ (Jana et al. 2000; Domenech et al. 2011), $\mathrm{N}_{2} \mathrm{H}_{4}$ (Yonezawa et al. 2001; Szilvia et al. 2007), ascorbic acid (Sun et al. 2007) and PEG (Luo et al. 2005; El-Houta et al. 2012). Over the past decade there has been an increased interest in the green chemistry (Roucoux et al. 2002; Raveendran et al. 2003). In the synthesis of metal nanoparticles by reduction of the corresponding metal ion salt solutions, there are three areas of opportunity to engage in green chemistry: (1) choice of solvent, (2) the reducing agent, and (3) the capping agent. There has also been increasing interest in identifying environmental friendly materials that are multifunctional (Nadagouda and Rajender 2008). Previously PdNPs were prepared through green methods using annona squamosa $\mathrm{L}$ peel extract (Roopana et al. 2012), banana peel extract (Ahok et al. 2010), cinnamom zeylanicum bark (Sathishkumar et al. 2009), broth of cinnamom camphora leaf (Yang et al. 2010) and gum acacia (Keerthi et al. 2011).

Xanthan gum (XG) is an extracellular polysaccharide secreted by the fermentation of the bacterium Xanthomonas campestris (Barrére et al. 1986). It is composed of pentasaccharide repeat units, comprising glucose, 
mannose, and glucuronic acid in the molar ratio 2.0:2.0:1.0 (Garcia-Ochoa et al. 2000). XG is used as a stabilizer, thickener and emulsifier. Due to its nontoxic and biocompatible properties, XG is widely used in food and pharmaceutical industries. In this paper, we report green synthesis of PdNPs using XG as both reducing and stabilizing agent without using any toxic chemicals. This reaction is carried out in an autoclave at a pressure of $15 \mathrm{psi}$ and at $120^{\circ} \mathrm{C}$ temperature for $10 \mathrm{~min}$. This method is more efficient and rapid synthesis process compared to previously reported green methods mentioned above (Roopana et al. 2012; Sathishkumar et al. 2009; Yang et al. 2010) for the synthesis of Pd nanoparticles.

Nitro phenols are important chemical materials which are widely used to manufacture explosives, drugs, insecticides and dyes, and also used as corrosion inhibitors of woods and rubber chemicals (Zhang et al. 2011). In addition, 4-nitrophenol (4-NP) is an intermediate in the synthesis of many organic compounds. Since 4-amino phenol (4-AP) is a potent industrial intermediate in the manufacturing of many analgesic (Sandip et al. 2010). Thus, being a common precursor material for 4-AP, a newer and cheaper method for catalytic hydrogenation of 4-NP is always in demand. The catalytic activities of PdNPs are tested on the reduction reaction of 4-NP by $\mathrm{NaBH}_{4}$. The reaction was studied by spectrophotometric methods. In the present study, we report the catalytic activity of green synthesized PdNPs towards 4-NP reduction.

\section{Experimental}

\section{Materials}

Palladium (II) chloride, $\mathrm{HCl}$, 4-NP, xanthan gum and sodium borohydride were purchased from S D Fine-chem Limited, Mumbai, India.

\section{Preparation of PdNPs}

Solutions were prepared with double distilled water. $0.0356 \mathrm{~g}$ of $\mathrm{PdCl}_{2}$ was accurately weighed and dissolved in $100 \mathrm{ml}$ of $\mathrm{HCl}(0.000413 \mathrm{M})$ to form an $\mathrm{H}_{2} \mathrm{PdCl}_{4}$ aqueous solution. An aliquot of $5 \mathrm{ml}$ of the aqueous $\mathrm{H}_{2} \mathrm{PdCl}_{4}$ solution was mixed with $5 \mathrm{ml}$ of a $0.2 \%$ aqueous solution of XG in a boiling tube. This reaction is carried out in an autoclave at a pressure of $15 \mathrm{psi}$ and at $120{ }^{\circ} \mathrm{C}$ temperature by 10 min time.

Catalytic activity

To monitor the homogeneous catalysis of 4-NP reduction by PdNPs, UNICAM UV-3600 UV-visible
Spectrophotometer (UV-Vis) (Thermo Spectronic) was used. To a 3-ml cuvette containing freshly prepared sodium borohydrate $(1 \mathrm{ml}, 15 \mathrm{mM})$ solution, $2 \mathrm{ml}$ of 4-NP $(0.2 \mathrm{mM})$ solution was added. After adding PdNPs stabilized in XG $(10 \mu \mathrm{l})$ solution, cuvette was shaken vigorously for mixing and kept in UV-Vis spectrophotometer to examine the reaction.

\section{Characterization}

To study the formation of palladium nanoparticles, the UV-Vis absorption spectra of the prepared solutions were recorded using an UNICAM UV-3600 spectrophotometer (Thermo Spectronic). Fourier transform infrared (FTIR) spectra of PdNPs stabilized in XG and XG alone were recorded in $\mathrm{KBr}$ pellets using FTIR spectrophotometer (Bruker optics, Germany) and the scan was performed in the range of $400-4,500 \mathrm{~cm}^{-1}$. X-ray diffraction (XRD) measurement of PdNPs stabilized in XG was carried out on X'pert Pro X-ray diffractometer (Panalytical B.V., Netherlands) operating at $40 \mathrm{kV}$ and a current of $30 \mathrm{~mA}$ at a scan rate of $0.388 \mathrm{~min}^{-1}$. The size and morphology of the nanoparticles were determined by TEM using TechnaiG2 under $200 \mathrm{kv}$.

\section{Results and discussion}

UV-visible analysis

Present studies focus on the synthesis of PdNPs using XG as reducing as well as stabilizing agent without using any toxic chemicals, which is purely green approach. The reaction was carried out in an autoclave at a pressure of 15 psi and at $120{ }^{\circ} \mathrm{C}$ temperature. The yellow colour reaction mixture was converted to the characteristic black colour after the autoclaving. The appearance of black colour indicates the formation of PdNPs nanoparticles (Keerthi et al. 2011). Figure 1 shows the absorption peak at $412 \mathrm{~nm}$, corresponding to mixture of $\mathrm{H}_{2} \mathrm{PdCl}_{4}$ and $\mathrm{XG}$. After autoclaving formation of PdNPs the peak at $412 \mathrm{~nm}$ disappears confirming the reduction of $\mathrm{Pd}$ (II) ions to $\mathrm{Pd}(0)$.

XRD analysis

The crystalline nature of resulting PdNPs synthesized can be seen in Fig. 2, in which all the peaks are clearly distinguishable. The broad peak at 39.73 is characteristics peak of the (111) indices of $\mathrm{Pd}(0)$ which is a face-centred cubic structure. Three other peaks at $2 \theta$ values of $46.09,67.82,81.52,86.87$ were observed corresponding to the major reflections of the (200), (220), (311), (222) crystal planes, respectively (Ramesh et al. 2012). Broadening of the diffraction peaks was observed 


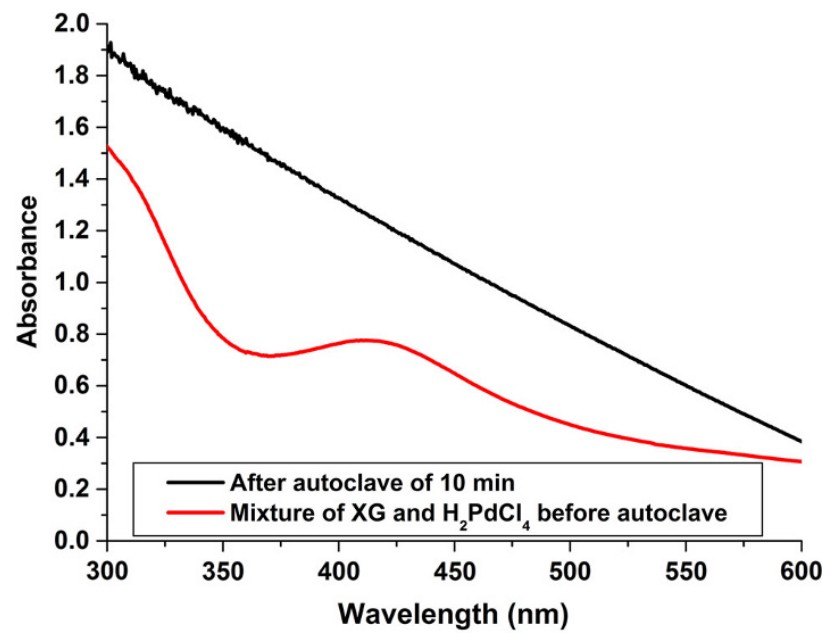

Fig. 1 UV-visible spectra of reaction mixture of $\mathrm{H}_{2} \mathrm{PdCl}_{4}$ and xanthan gum solution before and after autoclaving

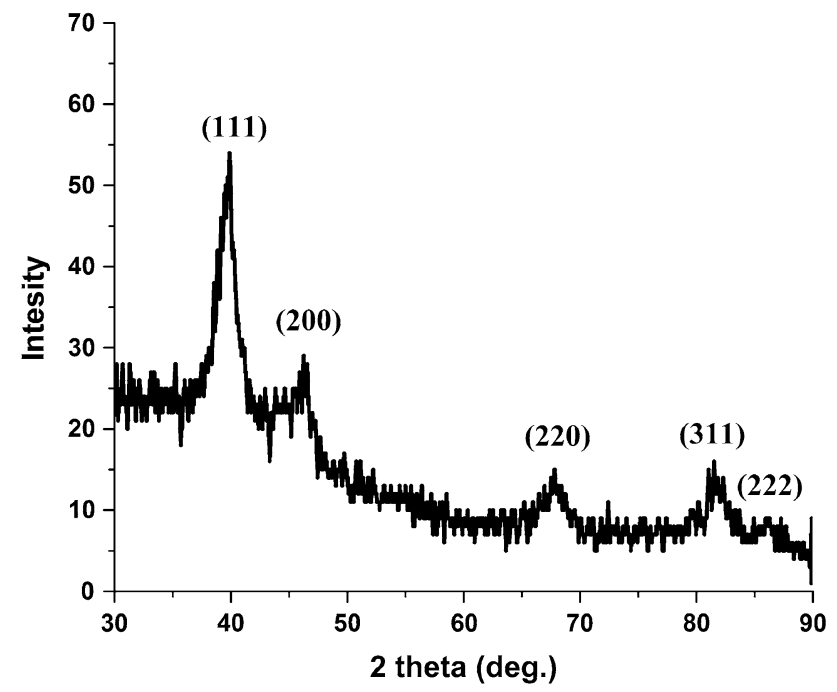

Fig. 2 Typical XRD pattern of the palladium nanoparticles stabilized in xanthan gum

owing to the effect of the nano-sized particles. The crystallite size of palladium nanoparticles is $10 \mathrm{~nm}$ which was calculated using peak broadening profile of (111) peak at $40^{\circ}$ using Sherrer's formula given below

$d=0.94(\lambda) / \beta \cos \theta$

where $\lambda$ is wavelength (1.5418 $\AA$ ) and $\beta$ is full-width halfmaximum (FWHM) of corresponding peak. The calculated crystallite size of the synthesized palladium nanoparticles is $10 \mathrm{~nm}$.

FTIR spectra analysis

FTIR analysis was used to identify the role of XG for the reduction and the capping of NPs surfaces. Figure 3 shows

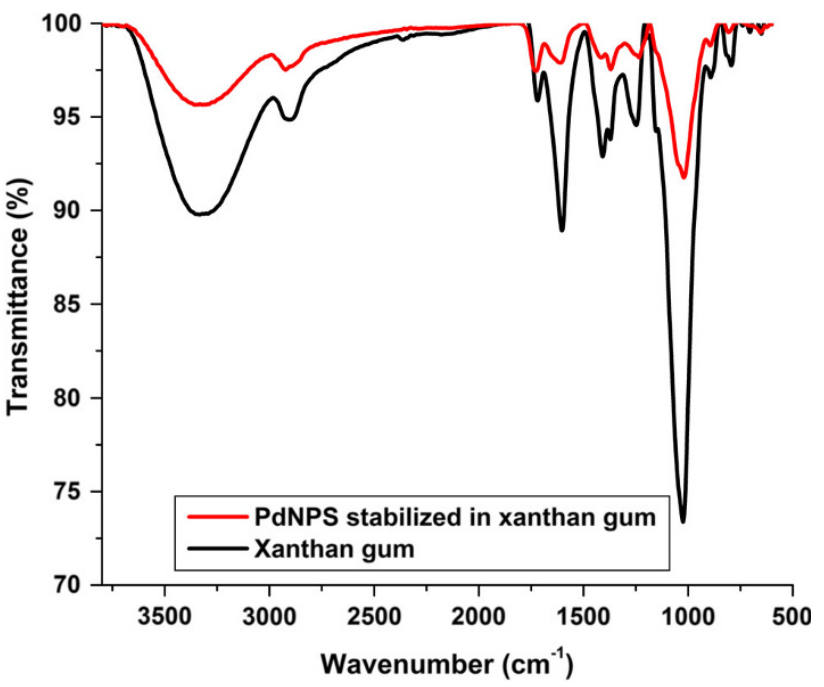

Fig. 3 FTIR spectra of palladium nanoparticles stabilized in xanthan gum and pure xanthan gum

FTIR spectrum of XG and PdNPs stabilized in XG. The major absorbance bands present in the spectrum of $\mathrm{XG}$ were at 3,334, 2,906, 1,719 and $1,603 \mathrm{~cm}^{-1}$. The broad bands observed at 3,334 and $2,906 \mathrm{~cm}^{-1}$ could be assigned to the stretching vibrations of $\mathrm{O}-\mathrm{H}$ groups and $-\mathrm{CH} 2,-\mathrm{CH} 3$ aliphatic groups in $\mathrm{XG}$. The bands found at 1,719 and $1,603 \mathrm{~cm}^{-1}$ could be due to the characteristic asymmetrical stretch of carboxylate group and carbonyl group. The peak at $1,024 \mathrm{~cm}^{-1}$ is due to the $\mathrm{C}-\mathrm{O}$ stretching vibration of alcoholic groups. The band at $3,334 \mathrm{~cm}^{-1}$ shifted to $3,320 \mathrm{~cm}^{-1}$ in the presence of PdNPs. These observations clearly show the interaction of $\mathrm{Pd}$ with the $\mathrm{OH}$ group of XG. The interactions among the resultant Pd nanoparticles and oxygen atoms of $\mathrm{O}-\mathrm{H},-\mathrm{COO}^{-}$and $-\mathrm{CO}$ become stronger. This can lead to corresponding changes both in the positions and in the strengths of FTIR spectra of XG. The variations in the shape and peak positions of the $-\mathrm{OH}$ stretching vibration, $-\mathrm{COO}^{-}$group, $-\mathrm{CO}$ group and $-\mathrm{OH}$ bending vibration at 3,334, 1,719, 1,603 and 1,024 $\mathrm{cm}^{-1}$, respectively, are observed, because of the contribution of $\mathrm{XG}$ towards the reduction and stabilization process (Venkatesham et al. 2014).

\section{TEM analysis}

The size distribution, shape and morphology of the PdNPs stabilized in XG were studied by high-resolution transmission electron microscopy. The TEM image of PdNPs stabilized in $\mathrm{XG}$ is shown in Fig. 4a. The TEM image shows that the PdNPs are spherical and are well distributed in the gum polymer matrix. To obtain size distributions of PdNPs, approximately 38 particles were counted and then converted into histograms. Figure $4 \mathrm{~b}$ presents a histogram 


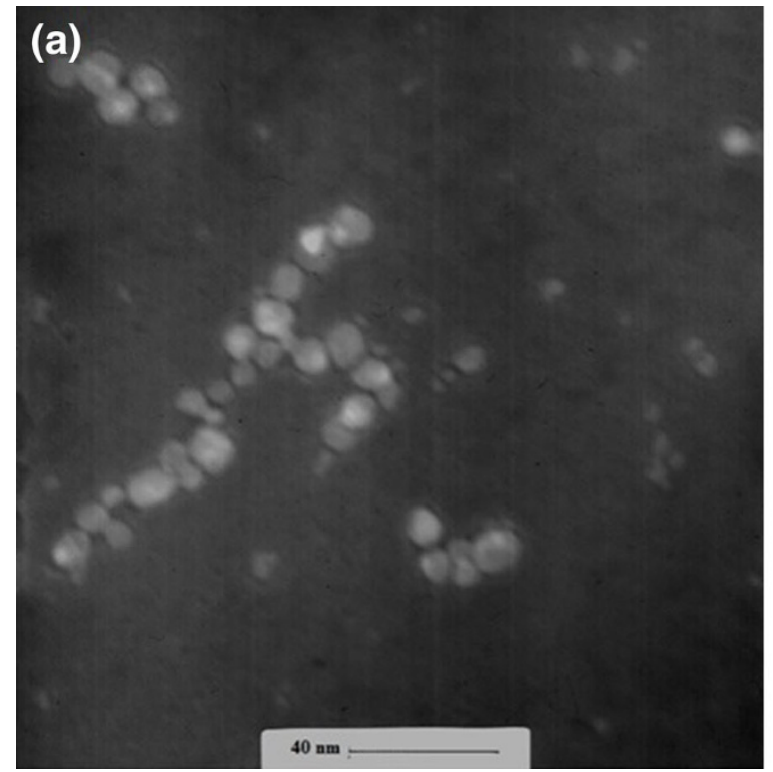

(b)

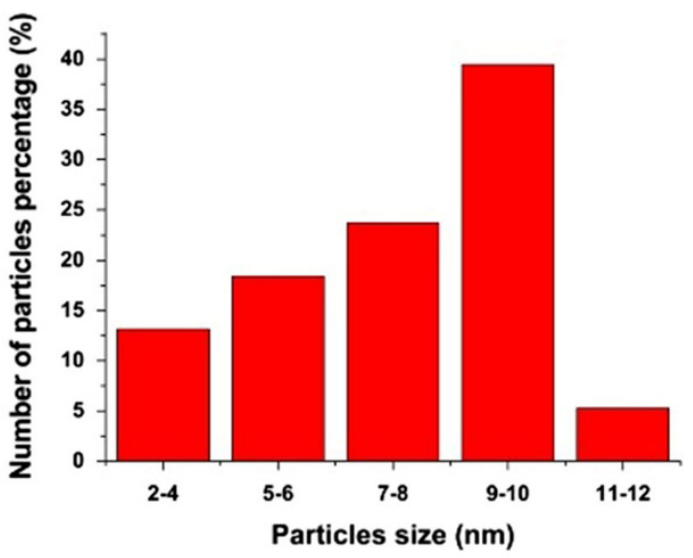

Fig. 4 a Typical TEM image of PdNPs in aqueous system using xanthan gum as reducing and stabilizing agent. b Histogram showing the size distribution of PdNPs

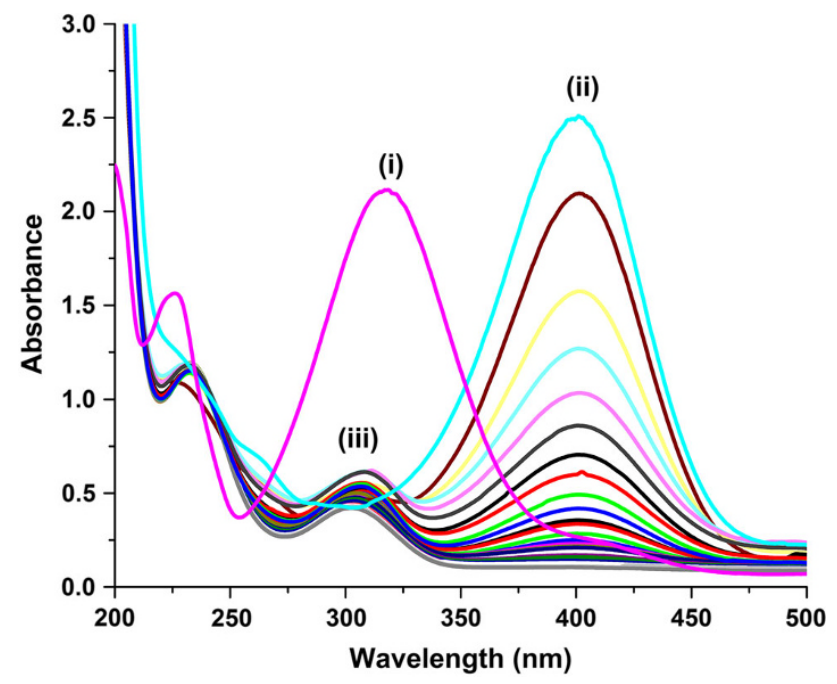

Fig. 5 UV-visible spectra of $i$ 4-nitrophenol, ii successive reduction of nitrophenolate ion with a time interval of $1 \mathrm{~min}$, and iii 4-aminophenol

of the particle size distribution of PdNPs. Most of the particles were in the size around $10 \mathrm{~nm}$.

\section{Catalytic reduction of 4-nitro phenol}

As Pd is recognized to be an excellent catalyst for hydrogenation reactions, we tried the PdNPs stabilized in XG for the reduction of 4-NP to 4-AP using borohydride. In a typical catalytic reaction, freshly prepared $\mathrm{NaBH}_{4}(1 \mathrm{ml}$, $15 \mathrm{mM}$ ) solution, taken in a 3-ml cuvette, was added to the aqueous 4-NP solution $(2 \mathrm{ml}, 0.2 \mathrm{mM})$. The 4-NP shows

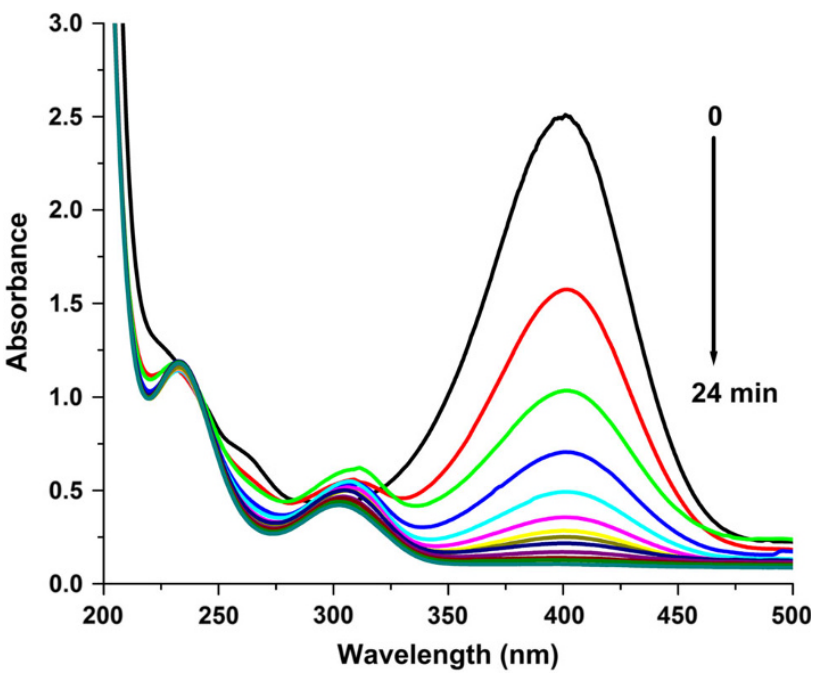

Fig. 6 The conversion percentage of 4-nitrophenol to 4-aminophenol with time

an absorbance peak at $317 \mathrm{~nm}$, which shifts to $400 \mathrm{~nm}$ (red shift) in the presence of $\mathrm{NaBH}_{4}$ due to the formation of 4-nitrophenolate ion (Fig. 5). In the absence of catalyst, the 4-nitrophenolate ions cannot be reduced further, even in the presence of strong reducing agent like $\mathrm{NaBH}_{4}$ (Venkatesham et al. 2014). However, immediately after adding $10 \mu \mathrm{l}$ of PdNPs to the reaction mixture, the intensity of the absorbance band at $400 \mathrm{~nm}$ successively decreased with increasing reaction time. At the same time a new band with increased absorbance intensity appears at $300 \mathrm{~nm}$, which is known to be due to absorption of 4-AP. 


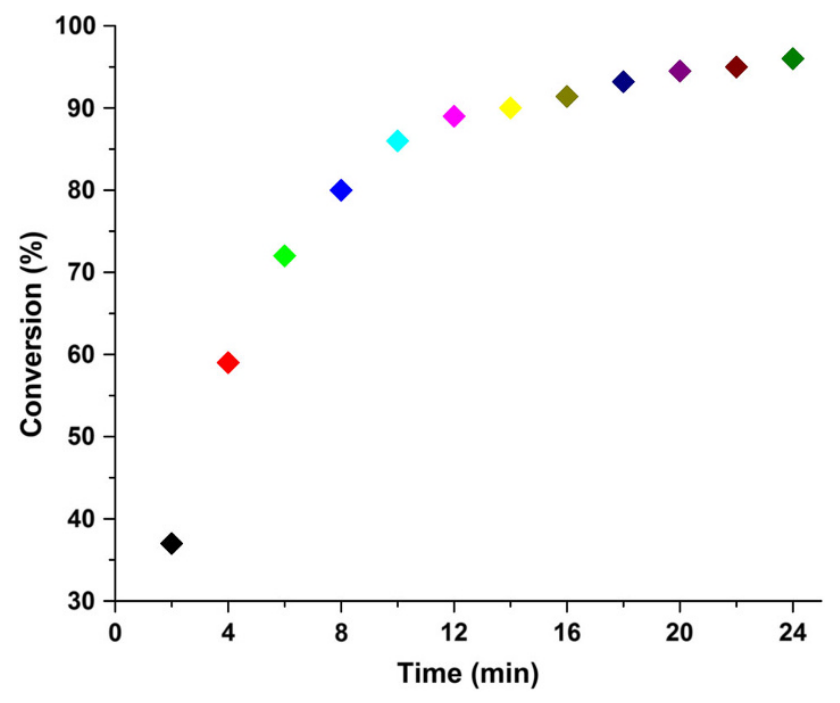

Fig. 7 Reduction of UV-Vis spectra of 4-nitrophenol using PdNPs stabilized in xanthan gum with time of interval $2 \mathrm{~min}$

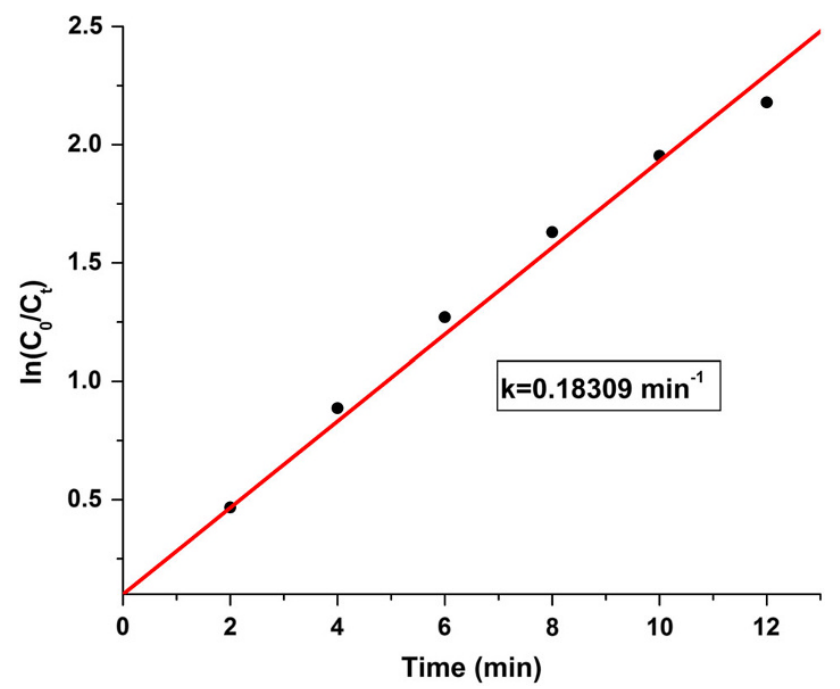

Fig. 8 The plot of $\ln C_{\mathrm{o}} / C_{t}$ versus time for the reduction of nitrophenol to aminophenol

The absorbance at time $t=0\left(A_{0}\right)$ and at $t\left(A_{t}\right)$ is proportional to the initial concentration $C_{0}$ and concentration at time $t\left(C_{t}\right)$ of 4-NP, respectively. The conversion percentage $(\alpha)$ of 4-NP to 4-aminophenol was calculated by the formula:

$\alpha=\frac{\left(C_{0}-C_{t}\right)}{C_{0}} 100$.

The conversion percentage of 4-NP to 4-AP is shown in Fig. 6.

It has been found that the reduction of 4-NP to 4-AP by sodium borohydride in the presence of PdNPs as catalyst follows pseudo first-order rate equation with respect to 4$\mathrm{NP}$; the concentration of sodium borohydride was too high as compared to 4-NP. So, concentration of sodium borohydride was considered constant throughout the reaction. UV-Vis spectra of successive reduction of 4-NP to 4aminophenol using PdNPs stabilized in XG with time interval of 2 min are shown in Fig. 7. The rate equation can be written as

$k=\frac{\ln \left(C_{0} / C_{t}\right)}{t}$.

Figure 8 shows a good linear correlation of $\ln \left(C_{\mathrm{o}} / C_{t}\right)$ versus time and the rate constant of the reaction is obtained as $0.18309 \mathrm{~min}^{-1}$ for PdNPs stabilized in XG.

\section{Conclusions}

The present study reports the green the synthesis, characterization and catalytic evaluation of PdNPs from aqueous $\mathrm{H}_{2} \mathrm{PdCl}_{4}$ solution using XG. The adapted method is compatible with green chemistry principles as the $\mathrm{XG}$ serves as a matrix for both reduction and stabilization of the PdNPs synthesized. The PdNPs stabilized in XG exhibited a very good catalytic activity and the kinetics of the reaction was found to be pseudo first order with respect to the 4-NP.

Acknowledgments The authors wish to thank the Coordinator, DBT-OU-ISLARE, Instrumentation Laboratory (Funded by UGC), Osmania University for providing facilities.

Open Access This article is distributed under the terms of the Creative Commons Attribution License which permits any use, distribution, and reproduction in any medium, provided the original author(s) and the source are credited.

\section{References}

Ahok B, Bhagyashree J, Ameeta RK, Smita Z (2010) Banana peel extract mediated novel route for the synthesis of palladium nanoparticles. Mat Lett 64:1951-1953

Barrére GC, Barber CE, Daniels MJ (1986) Molecular cloning of genes involved in the production of the extracellular polysaccharide xanthan by Xanthomonas campestris. Intern $\mathrm{J}$ Bio Macromol 8:372-374

Domenech B, Munoz M, Muraviev DN, Macanas J (2011) Polymerstabilized palladium nanoparticles for catalytic membranes: ad hoc polymer fabrication. Nanoscale Res Lett 6:406

El-Houta SE, Killab HM, Ibrahima IA, Harraz FA (2012) Palladium nanoparticles stabilized by polyethylene glycol: efficient, recyclable catalyst for hydrogenation of styrene and nitrobenzene. J Catal 286:184-192

Garcia-Ochoa F, Santos VE, Casas JA, Gomez E (2000) Xanthan gum: production, recovery, and properties. Biotech Adv 18:549-579

Jana NR, Wang ZL, Pal T (2000) Redox catalytic properties of palladium nanoparticles: surfactant and electron donor-acceptor effects. Langmuir 16:2457-2463

Karimi B, Enders D (2006) New N-heterocyclic carbene palladium complex/ionic liquid matrix immobilized on silica: application

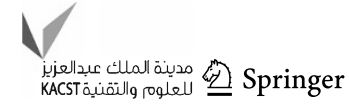


as recoverable catalyst for the heck reaction. Org Lett 8:1237-1240

Keerthi DD, Veera PSV, Harith R, Samba SK, Radhika P, Sreedhar B (2011) Gum acacia as a facile reducing, stabilizing, and templating agent for palladium nanoparticles. J Appl Polym Sci 121:1765-1773

Klingensmith LM, Leadbeater NE (2003) Ligand-free palladium catalysis of aryl coupling reactions facilitated by grinding. Tetrahedron Lett 44:765-768

Luo C, Zhang Y, Wang Y (2005) Palladium nanoparticles in poly (ethylene glycol): the efficient and recyclable catalyst for heck reaction. J Mol Catal A 229:7-12

Nadagouda MN, Rajender SV (2008) Green synthesis of silver and palladium nanoparticles at room temperature using coffee and tea extract. Green Chem 10:859-862

Narayanan R, El-Sayed MA (2005) FTIR study of the mode of binding of the reactants on the Pd nanoparticle surface during the catalysis of the Suzuki reaction. J Phys Chem B 109:4357-4360

Prashant KJ, Kyeong SL, El-Sayed IH, El-Sayed MA (2006) Calculated absorption and scattering properties of gold nanoparticles of different size, shape, and composition: applications in biological imaging and biomedicine. J Phys Chem B 110:7238-7248

Ramesh KP, Vivekanandhan S, Manjusri M, Amar KM, Satyanarayana N (2012) Soybean (Glycine max) leaf extract based green synthesis of palladium nanoparticles. J Biomater Nanobiotechnol 3:14-19

Raveendran P, Fu J, Wallen SL (2003) Completely "Green" synthesis and stabilization of metal nanoparticles. J Am Chem Soc 125:13940-13941

Roopana SM, Bharathi A, Rajendran K, Khanna VG, Prabhakarn A (2012) Acaricidal, insecticidal, and larvicidal efficacy of aqueous extract of Annona squamosa L peel as biomaterial for the reduction of palladium salts into nanoparticles. Collid Surf B Biointerfaces 92:209-212

Rosi NL, Mirkin CA (2005) Nanostructures in biodiagnostics. Chem Rev 105:1547-1562

Roucoux A, Schulz J, Patin H (2002) Reduced transition metal colloids: a novel family of reusable catalysts? Chem Rev 102:3757-3778
Salata OV (2004) Applications of nanoparticles in biology and medicine. J Nanobiotechnol 2:3

Sandip S, Anjaliv P, Subrata K, Soumen B, Tarasankar P (2010) Photochemical green synthesis of calcium-alginate-stabilized Ag and $\mathrm{Au}$ nanoparticles and their catalytic application to 4-nitrophenol reduction. Langmuir 26:2885

Sathishkumar M, Sneha K, Kwak IS, Mao J, Tripathy SJ, Yun YS (2009) Phyto-crystallization of palladium through reduction process using cinnamom zeylanicum bark extract. J Hazard Mater 171:400-404

Sun Y, Zhang LH, Zhou H, Zhu Y, Sutter E, Ji Y, Rafailovich MH, Sokolov JC (2007) Seedless and templateless synthesis of rectangular palladium nanoparticles. Chem Mater 19:2065-2070

Szilvia P, Pi Rita, Imre D (2007) Formation and stabilization of noble metal nanoparticles croat. Chem Acta 80:493-502

Venkatesham M, Ayodhya D, Madhusudhan A, Veera BN, Veerabhadram G (2014) A novel green one-step synthesis of silver nanoparticles using chitosan: catalytic activity and antimicrobial studies. Appl Nanosci 4:113-119

Wilson OM, Knecht MR, Garcia-Martinez JC, Crooks RM (2006) Effect of Pd nanoparticle size on the catalytic hydrogenation of allyl alcohol. J Am Chem Soc 128:4510-4511

Yang M, Yang Y, Liu Y, Shen G, Yu R (2006) Platinum nanoparticles-doped sol-gel/carbon nanotubes composite electrochemical sensors and biosensors. Biosens Bioelectron 21:1125-1131

Yang X, Li Q, Wang H, Huang J, Lin L, Wang W, Sun D, Su Y, Opiyo JB, Hong L, Wang Y, He N, Jia L (2010) Green synthesis of palladium nanoparticles using broth of Cinnamomum camphora leaf. J Nano par Res 12:1589-1598

Yonezawa T, Imamura K, Kimizuka N (2001) Direct preparation and size control of palladium nanoparticle hydrosols by watersoluble isocyanide ligands. Langmuir 17:4701-4703

Zhang P, Shao C, Zhang Z, Zhang M, Mu J, Guo Z, Liu Y (2011) In situ assembly of well-dispersed Ag nanoparticles (AgNPs) on electrospun carbon nanofibers (CNFs) for catalytic reduction of 4-nitrophenol. Nanoscale 3:3357 